\title{
Telaprevir for Previously Untreated Chronic Hepatitis C Virus Infection
}

\begin{abstract}
Ira M. Jacobson, M.D., John G. McHutchison, M.D., Geoffrey Dusheiko, M.D., Adrian M. Di Bisceglie, M.D., K. Rajender Reddy, M.D., Natalie H. Bzowej, M.D., Patrick Marcellin, M.D., Andrew J. Muir, M.D., Peter Ferenci, M.D., Robert Flisiak, M.D., Jacob George, M.D., Mario Rizzetto, M.D., Daniel Shouval, M.D., Ricard Sola, M.D., Ruben A. Terg, M.D., Eric M. Yoshida, M.D., Nathalie Adda, M.D., Leif Bengtsson, B.Sc., Abdul J. Sankoh, Ph.D., Tara L. Kieffer, Ph.D., Shelley George, M.D., Robert S. Kauffman, M.D., Ph.D., and Stefan Zeuzem M.D., for the ADVANCE Study Team*
\end{abstract}

\section{ABSTRACT}

\section{BACKGROUND}

In phase 2 trials, telaprevir, a hepatitis $\mathrm{C}$ virus (HCV) genotype 1 protease inhibitor, in combination with peginterferon-ribavirin, as compared with peginterferon-ribavirin alone, has shown improved efficacy, with potential for shortening the duration of treatment in a majority of patients.

\section{METHODS}

In this international, phase 3, randomized, double-blind, placebo-controlled trial, we assigned 1088 patients with HCV genotype 1 infection who had not received previous treatment for the infection to one of three groups: a group receiving telaprevir combined with peginterferon alfa-2a and ribavirin for 12 weeks (T12PR group), followed by peginterferon-ribavirin alone for 12 weeks if HCV RNA was undetectable at weeks 4 and 12 or for 36 weeks if HCV RNA was detectable at either time point; a group receiving telaprevir with peginterferon-ribavirin for 8 weeks and placebo with peginterferon-ribavirin for 4 weeks (T8PR group), followed by 12 or 36 weeks of peginterferon-ribavirin on the basis of the same HCV RNA criteria; or a group receiving placebo with peginterferon-ribavirin for 12 weeks, followed by 36 weeks of peginterferon-ribavirin (PR group). The primary end point was the proportion of patients who had undetectable plasma HCV RNA 24 weeks after the last planned dose of study treatment (sustained virologic response).

RESULTS

Significantly more patients in the T12PR or T8PR group than in the PR group had a sustained virologic response (75\% and 69\%, respectively, vs. $44 \%$; $\mathrm{P}<0.001$ for the comparison of the T12PR or T8PR group with the PR group). A total of $58 \%$ of the patients treated with telaprevir were eligible to receive 24 weeks of total treatment. Anemia, gastrointestinal side effects, and skin rashes occurred at a higher incidence among patients receiving telaprevir than among those receiving peginterferon-ribavirin alone. The overall rate of discontinuation of the treatment regimen owing to adverse events was $10 \%$ in the T12PR and T8PR groups and $7 \%$ in the PR group.

\section{CONCLUSIONS}

Telaprevir with peginterferon-ribavirin, as compared with peginterferon-ribavirin alone, was associated with significantly improved rates of sustained virologic response in patients with HCV genotype 1 infection who had not received previous treatment, with only 24 weeks of therapy administered in the majority of patients. (Funded by Vertex Pharmaceuticals and Tibotec; ADVANCE ClinicalTrials.gov number, NCT00627926.)
From the Weill Cornell Medical College and Center for the Study of Hepatitis C, New York (I.M.J.); Duke Clinical Research Institute and Division of Gastroenterology, Duke University Medical Center, Durham, NC (J.G.M., A.J.M.); Royal Free Hospital, Centre for Hepatology, London (G.D.); Saint Louis University School of Medicine, St. Louis (A.M.D.B.); Division of Gastroenterology, University of Pennsylvania, Philadelphia (K.R.R.); California Pacific Medical Center, San Francisco (N.H.B.); Hôpital Beaujon, Service d'Hépatologie and INSERM CRB3, Clichy, France (P.M.); University of Vienna, Vienna (P.F.); Department of Infectious Diseases and Hepatology, Medical University of Bialystok, Bialystok, Poland (R.F.); Storr Liver Unit, Westmead Millennium Institute for Medical Research and Westmead Hospital, University of Sydney, Westmead, NSW, Australia (J.G.); Department of Gastroenterology, University of Turin, Turin, Italy (M.R.); Liver Unit, Hadassah-Hebrew University Hospital, Jerusalem, Israel (D.S.); Hospital del Mar, Institut Municipal d'Investigació Médica, Universitat Autónoma de Barcelona, Barcelona (R.S.); Hospital de Gastroenterología Dr. Bonorino Udaondo, Buenos Aires (R.A.T.); University of British Columbia and Vancouver General Hospital, Vancouver, BC, Canada (E.M.Y.); Vertex Pharmaceuticals, Cambridge, MA (N.A., L.B., A.J.S., T.L.K., S.G., R.S.K.); and Department of Internal Medicine, Johann Wolfgang Goethe University Medical Center, Frankfurt am Main, Germany (S.Z.). Address reprint requests to Dr. Jacobson at the Weill Cornell Medical College and Center for the Study of Hepatitis C, 1305 York Ave., New York, NY 10021, or atimj2001@med.cornell.edu.

*The members of the ADVANCE Study Team are listed in the Supplementary Appendix, available at NEJM.org.

N Engl J Med 2011;364:2405-16.

Copyright (c) 2011 Massachusetts Medical Society. 
P ATIENTS WITH CHRONIC HEPATITIS C VIRUS (HCV) infection are at risk for progressive hepatic fibrosis, cirrhosis, portal hypertension, hepatic failure, and hepatocellular carcinoma..$^{1-4}$ For the past decade, treatment with pegylated interferon (peginterferon alfa) and ribavirin has been associated with rates of sustained virologic response of 40 to $50 \%$ among patients with HCV genotype 1 who had received no previous treatment..$^{5-7}$ At least 48 weeks of treatment is required for most of these patients, and toxic effects may limit the extent of treatment in some patients..$^{5-7}$

Telaprevir, a linear peptidomimetic HCV NS3/4A serine protease inhibitor, was associated with substantial improvements in response rates in phase 2 studies when it was combined with peginterferonribavirin. ${ }^{8-10}$ Moreover, high rates of early viral suppression and low rates of relapse after cessation of telaprevir therapy suggested that therapy could potentially be shortened to 24 weeks in patients who have a rapid virologic response - that is, patients in whom HCV RNA is undetectable at week 4 of treatment. $^{8-10}$ A phase 3 study was conducted to evaluate the efficacy and safety of telaprevir-based therapy, administered in a regimen that was guided by the patient's response, among patients who had received no previous treatment for $\mathrm{HCV}$ infection.

\section{METHODS}

\section{PATIENTS}

We enrolled patients at 123 international sites. Eligible patients were 18 to 70 years of age and had $\mathrm{HCV}$ genotype 1 infection with evidence of chronic hepatitis, as confirmed by means of a liver biopsy within 1 year before screening for the study; patients with compensated liver cirrhosis were eligible. Additional eligibility criteria included seronegativity for hepatitis B surface antigen, and the absence of antibodies against human immunodeficiency virus types 1 and 2, absolute neutrophil counts of 1500 or more per cubic millimeter, platelet counts of 90,000 or more per cubic millimeter, and hemoglobin levels of at least $12 \mathrm{~g}$ per deciliter in the case of women or $13 \mathrm{~g}$ per deciliter in the case of men. Patients were excluded if they had decompensated liver disease, liver disease from other causes, or hepatocellular carcinoma.

\section{STUDY DESIGN}

This study was a phase 3, randomized, double-blind trial, placebo-controlled for telaprevir. Patients were stratified according to genotype 1 subtype (a, b, or unknown) and baseline viral load (HCV RNA $<800,000$ IU per milliliter or $\geq 800,000$ IU per milliliter) and were randomly assigned to one of three treatment groups. The study was designed to evaluate two regimens of telaprevir (Vertex Pharmaceuticals) of different durations, combined with peginterferon alfa-2a (Pegasys, Roche) and ribavirin (Copegus, Roche), as compared with a regimen of peginterferon alfa-2a and ribavirin alone. The total duration of treatment was either 24 or 48 weeks. During the first 12 weeks, patients assigned to one of the telaprevir groups received telaprevir and peginterferon-ribavirin either for the entire 12 weeks (T12PR group) or for 8 weeks followed by 4 weeks of placebo and peginterferonribavirin (T8PR group). Patients in the T12PR and T8PR groups who met the criteria for an extended rapid virologic response (defined as undetectable HCV RNA at weeks 4 and 12) received 12 additional weeks of treatment with peginterferonribavirin alone, for a total treatment period of 24 weeks. Patients in the T12PR and T8PR groups who had detectable HCV RNA either at week 4 or at week 12 received 36 additional weeks of treatment with peginterferon-ribavirin, for a total treatment period of 48 weeks. The group receiving peginterferon alfa-2a and ribavirin alone (PR group) received placebo plus peginterferonribavirin for 12 weeks, followed by peginterferonribavirin alone for 36 additional weeks. Telaprevir was administered orally at a dose of $750 \mathrm{mg}$ every 8 hours with food, peginterferon alfa-2a by subcutaneous injection at a dose of $180 \mu \mathrm{g}$ per week, and ribavirin orally at a dose of $1000 \mathrm{mg}$ per day (in patients who weighed less than $75 \mathrm{~kg}$ ) or $1200 \mathrm{mg}$ per day (in patients who weighed $75 \mathrm{~kg}$ or more).

Stopping rules were implemented to prevent the continuation of treatment in patients who did not have an adequate response. Patients receiving telaprevir who had HCV RNA levels greater than 1000 IU per milliliter at week 4 discontinued telaprevir but continued peginterferon-ribavirin. All patients with less than a $2 \log _{10}$ decrease from baseline in HCV RNA levels at week 12 discontinued treatment. Patients discontinued treatment if HCV RNA was confirmed to be detectable at any time between weeks 24 and 40 .

\section{STUDY OVERSIGHT}

The protocol was designed by Vertex Pharmaceuticals and Tibotec in collaboration with one of the 
academic authors. The protocol, which is available with the full text of this article at NEJM.org, was approved by an independent or institutional review board at each participating center, and all patients provided written informed consent before participating in study-related activities. The corresponding author prepared the first draft of the manuscript and made the decision to submit the manuscript for publication, and all the authors, together with an employee of the sponsor, assisted in the revision of subsequent drafts. All the authors reviewed and approved the final draft of the manuscript and assume responsibility for the accuracy and completeness of the data and data analyses and for the fidelity of the study to the trial protocol.

\section{EFFICACY ASSESSMENTS}

Plasma HCV RNA levels were measured with the use of the COBAS TaqMan HCV RNA assay, version 2.0 (Roche), with a lower limit of quantification of $25 \mathrm{IU}$ per milliliter and a lower limit of detection of $10 \mathrm{IU}$ per milliliter. The lower limit of detection was used in the determination of extended rapid virologic response. HCV RNA levels were measured on day 1 and at weeks $1,2,3,4,6,8$, $10,12,16,20,24,28,36,40$, and 48; at follow-up visits 4 weeks after the end of treatment; and at weeks 60 and 72 .

\section{EVALUATION OF HCV SEQUENCE}

Blood samples were obtained for viral sequencing at baseline and at every treatment and follow-up study visit. Sequencing samples from all the patients were analyzed at baseline, and those from patients who did not meet the criteria for a sustained virologic response were analyzed at all post-baseline time points at which HCV RNA levels were above the limit of detection of the sequencing assay (approximately 1000 IU per milliliter). HCV RNA was isolated from the plasma and amplified by reverse-transcriptase polymerase chain reaction, and the NS3/4A region of the HCV genome was analyzed with the use of population sequencing.

\section{SAFETY ASSESSMENTS}

Chemical and hematologic assessments were performed at the same time as the efficacy assessments during the treatment period and at 4 weeks after the last dose of the study drug was administered. Data on adverse events were collected at each treatment visit and at the safety follow-up assessment. Full physical examinations were performed at the screening visit and at the safety follow-up assessment. Physical examinations were performed as needed for the assessment and treatment of symptoms during treatment visits.

Because an increased rate of rash had been observed in phase 2 studies, ${ }^{8-10}$ guidance regarding the grading and management of rash was incorporated into this study. Rash was classified as grade 1 (mild, localized), grade 2 (moderate, with a diffuse skin eruption involving up to $50 \%$ of the body surface), or grade 3 (severe, involving more than $50 \%$ of the body surface, or rash with the appearance of substantial systemic signs or symptoms). If a progressive grade 2 rash or any grade 3 rash developed, telaprevir or placebo was to be discontinued but the patient would continue to receive peginterferon-ribavirin. If the rash worsened within 7 days after discontinuation of telaprevir or placebo, ribavirin (with or without peginterferon) was to be discontinued (sequential discontinuation).

Anemia was to be managed by means of reductions in the dose of ribavirin in accordance with the product labeling. Erythropoietin-stimulating agents were prohibited according to the final amended study protocol, as were reductions in the dose of telaprevir. If ribavirin was discontinued owing to anemia, discontinuation of telaprevir (or placebo) was required.

An independent data and safety monitoring committee conducted regular planned reviews of the safety data to evaluate safety and side effects of the study regimens. The analyses and preparation of the safety data for each review of the committee were performed by a statistical group that was independent of the sponsor (Parexel International). No interim analyses were planned or conducted.

\section{END POINTS}

The primary end point was the proportion of patients who had undetectable plasma HCV RNA 24 weeks after the last planned dose of study treatment (sustained virologic response). Secondary efficacy end points included the proportion of patients with undetectable HCV RNA at week 72; at week 4 , week 12 , or both weeks 4 and 12; at the end of treatment; and 12 weeks after the last planned dose of treatment. A patient was considered to have had a relapse if he or she had undetectable HCV RNA levels at the end of the treatment period but had confirmed detectable HCV 
RNA levels sometime between the end of treatment and 24 weeks after the last study dose.

\section{STATISTICAL ANALYSIS}

The analysis of the primary end point was based on a logistic-regression model, with sustained virologic response as the dependent variable and treatment, genotype 1 subtype, and baseline HCV RNA plasma level as factors. The primary end point was also evaluated by an analysis of the consistency of the treatment effect in prespecified subgroups according to 10 baseline variables (see the statistical analysis plan provided with the protocol at NEJM.org). We estimated that with a sample size of 350 patients in each treatment group, the study would have $92 \%$ power to show a significant difference among the treatments, with the use of a two-sided, continuity-corrected chi-square test, at an overall significance level of $5 \%$ (adjusted for multiple comparisons), assuming a $50 \%$ response rate in the control (PR) group and a 64\% response rate in a telaprevir group. Efficacy and safety analyses included data from all patients who underwent random assignment and received at least one dose of any study drug.

RESULTS

\section{STUDY PATIENTS}

Of the 1095 patients enrolled in the study, 1088 received at least one dose of a study drug and were included in the data set for the full analysis (Fig. 1). Patients were well balanced with respect to major baseline demographic and disease characteristics (Table 1). A total of $58 \%$ of the patients were men, $9 \%$ were black, $11 \%$ were Hispanic, and $21 \%$ had bridging fibrosis or cirrhosis.

\section{EFFICACY}

A significantly greater proportion of patients in each of the two groups receiving telaprevir than in the group receiving peginterferon-ribavirin alone met the criteria for a sustained virologic response (undetectable plasma HCV RNA 24 weeks after the last planned dose of study treatment): $75 \%$ in the T12PR group and $69 \%$ in the T8PR group, as compared with $44 \%$ in the PR group $(\mathrm{P}<0.001$ for the comparison of either telaprevir group with the PR group) (Table 2). A total of $73 \%$ of patients in the T12PR group, $67 \%$ in the T8PR group, and $44 \%$ in the PR group had undetectable HCV RNA 72 weeks after starting treatment $(\mathrm{P}<0.001$ for the comparison of either telaprevir group with the PR group); $68 \%, 66 \%$, and $9 \%$ in the three groups, respectively, had undetectable HCV RNA at week 4 (rapid virologic response); and 58\%, 57\%, and $8 \%$ in the three groups, respectively, had undetectable HCV RNA at weeks 4 and 12 (extended rapid virologic response). Among the patients with extended rapid virologic response assigned to receive a total of 24 weeks of therapy, $89 \%$ in the T12PR group and $83 \%$ in the T8PR group met the criteria for sustained virologic response. Mean HCV RNA levels during treatment are shown in Figure 2 in the Supplementary Appendix, available at NEJM.org.

Analyses of subgroups according to various characteristics showed that there were higher rates of sustained virologic response with telaprevir than with peginterferon-ribavirin alone (Fig. 2, and Fig. 1 in the Supplementary Appendix). A sustained virologic response occurred in $71 \%$ of the patients with HCV genotype 1a and $79 \%$ with genotype $1 \mathrm{~b}$ in the T12PR group, in $66 \%$ and $74 \%$ of the patients with genotype $1 \mathrm{a}$ and genotype $1 \mathrm{~b}$, respectively, in the T8PR group, and in $41 \%$ and $48 \%$ in of the patients with genotype $1 \mathrm{a}$ and genotype $1 \mathrm{~b}$, respectively, in the PR group. Among black patients, $62 \%$ in the T12PR group and $58 \%$ in the T8PR group had a sustained virologic response, as compared with $25 \%$ in the PR group. Among patients with HCV RNA levels of 800,000 IU per milliliter or more at baseline, those who received telaprevir had a higher rate of response than did those who received peginterferon-ribavirin alone (74\% of the patients in the T12PR group and 66\% in the T8PR group vs. $36 \%$ in the PR group). Among patients with bridging fibrosis or cirrhosis, $62 \%$ of patients in the T12PR group and $53 \%$ in the T8PR group, as compared with $33 \%$ in the PR group, had a sustained virologic response.

Among patients who had undetectable HCV RNA levels after the last dose of study treatment, relapse occurred in $9 \%$ in the T12PR group, $9 \%$ in the T8PR group, and $28 \%$ in the PR group. Recipients of a telaprevir-based regimen who were assigned to 24 weeks of treatment and who met the criteria for sustained virologic response were assessed for relapse beyond 24 weeks after the last study dose. One of 357 patients evaluated through week $72(<1 \%)$ had a confirmed late relapse after early discontinuation of the T8PR regimen at week 12. Three others had detectable HCV RNA 
below 25 IU per milliliter; in 2 of these patients, HCV RNA was subsequently undetectable, and in 1 , there was no available confirmation of $\mathrm{HCV}$ RNA level. One patient had HCV RNA of more than 20 million IU per milliliter, but the sequencing assay (limit of detection, approximately 1000 IU per milliliter) was unsuccessful, raising the possibility of a sample error.

A patient was considered to have virologic fail- ure during the treatment period if he or she met the criteria for a stopping rule, had HCV RNA greater than 1000 IU per milliliter at week 12 even if the HCV RNA decline was greater than $2 \log _{10}$, or had detectable HCV RNA at the end of treatment (week 24 or 48). The rate of virologic failure during the treatment period was lower among patients who received telaprevir than among those who received peginterferon-ribavirin alone $(8 \%$ in

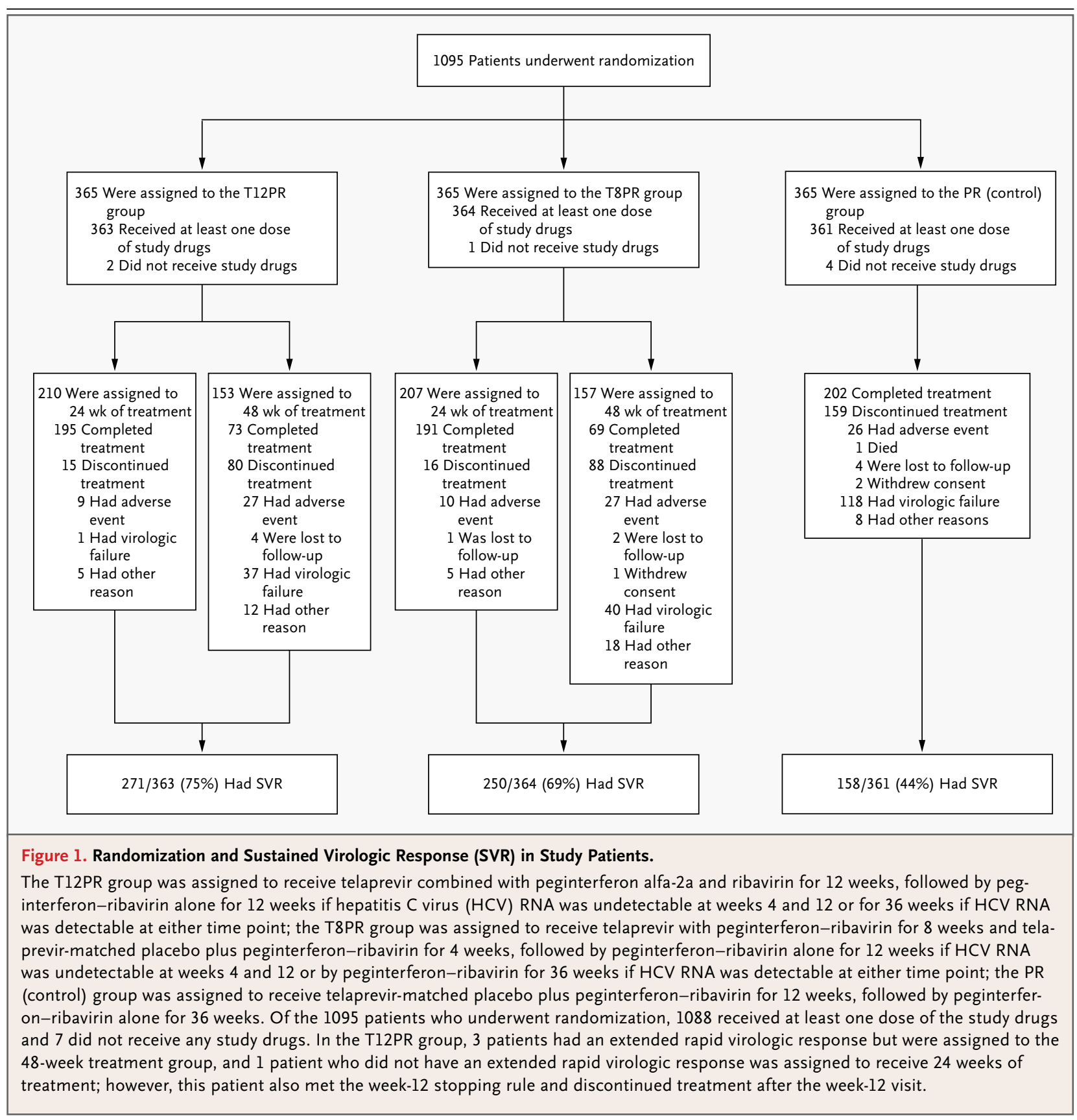


the T12PR group and $13 \%$ in the T8PR group, vs. $32 \%$ in the PR group). There were similar rates of virologic failure in the T12PR group and the T8PR group during the telaprevir treatment phase up to week 12 (3\%), and the virologic failure was attributable primarily to higher-level resistant variants of the HCV virus (e.g., V36M+R155K). After week 12 , the rates of virologic failure were higher in the T8PR group than in the T12PR group ( $10 \%$ vs. $5 \%$ ), with more frequent emergence of wild-type and lower-level resistant variants (e.g., V36A/M, T54A, and $\mathrm{R} 155 \mathrm{~K} / \mathrm{T}) .{ }^{11}$ Virologic failure was more common among patients with HCV genotype 1a infection than among those with HCV genotype $1 \mathrm{~b}$ infection.

\section{SAFETY}

The incidence of gastrointestinal disorders (nausea and diarrhea), pruritus, rash, and anemia was at least 10 percentage points higher in either of the telaprevir groups than in the PR group (Table 3). Other common adverse events are shown in Table 1 in the Supplementary Appendix. A total of $10 \%$ of the patients in the T12PR group, $10 \%$ in the T8PR group, and $7 \%$ in the PR group discontinued all treatment at some time during the study owing to adverse events (Table 2 in the Supplementary Appendix), whereas $7 \%, 8 \%$, and $4 \%$ of the patients in the three groups, respectively, discontinued all treatment during the telaprevir (or placebo) phase of the study owing to adverse events. The rate of

\begin{tabular}{|c|c|c|c|}
\hline Characteristic & $\begin{array}{c}\text { T12PR } \\
(\mathrm{N}=363)\end{array}$ & $\begin{array}{c}\text { T8PR } \\
(\mathrm{N}=364)\end{array}$ & $\begin{array}{c}\mathrm{PR} \\
(\mathrm{N}=361)\end{array}$ \\
\hline \multicolumn{4}{|l|}{ Age $-y r$} \\
\hline Median & 49 & 49 & 49 \\
\hline Range & $19-69$ & $19-68$ & $18-69$ \\
\hline \multicolumn{4}{|l|}{ Body-mass index广 } \\
\hline Median & 25.7 & 26.2 & 26.4 \\
\hline Range & $18-47$ & $17-46$ & $17-48$ \\
\hline \multicolumn{4}{|l|}{ Distribution — no. (\%) } \\
\hline$<25$ & $155(43)$ & $145(40)$ & $130(36)$ \\
\hline 25 to $<30$ & $129(36)$ & $131(36)$ & $144(40)$ \\
\hline$\geq 30$ & $77(21)$ & $86(24)$ & $87(24)$ \\
\hline Male sex — no. (\%) & $214(59)$ & $211(58)$ & $211(58)$ \\
\hline \multicolumn{4}{|l|}{ Race - no. $(\%) \ddagger$} \\
\hline White & $325(90)$ & $315(87)$ & $318(88)$ \\
\hline Black & $26(7)$ & $40(11)$ & $28(8)$ \\
\hline Asian & $5(1)$ & $5(1)$ & $10(3)$ \\
\hline Other & $7(2)$ & $4(1)$ & $5(1)$ \\
\hline \multicolumn{4}{|l|}{ Ethnic group - no. (\%) $¥$} \\
\hline Hispanic & $35(10)$ & $44(12)$ & $38(11)$ \\
\hline Non-Hispanic & $328(90)$ & $320(88)$ & $323(89)$ \\
\hline Alanine aminotransferase $-I U /$ liter & $84 \pm 69$ & $80 \pm 62$ & $88 \pm 67$ \\
\hline Total bilirubin $-\mu \mathrm{mol} /$ liter $\int$ & $10 \pm 5$ & $9 \pm 4$ & $9 \pm 4$ \\
\hline Serum albumin $-g /$ liter & $45 \pm 3$ & $44 \pm 3$ & $44 \pm 3$ \\
\hline Platelet count $-\times 10^{-9} /$ liter & $250 \pm 73$ & $236 \pm 65$ & $243 \pm 70$ \\
\hline \multicolumn{4}{|l|}{ HCV subtype — no. (\%) q } \\
\hline la & $213(59)$ & $210(58)$ & $208(58)$ \\
\hline $1 b$ & $149(41)$ & $151(41)$ & $151(42)$ \\
\hline Unknown & $1(<1)$ & $3(1)$ & $2(1)$ \\
\hline
\end{tabular}




\begin{tabular}{|lccc|}
\hline Table 1. (Continued.) & & & \\
\hline \\
Characteristic & T12PR & T8PR & PR \\
HCV RNA - $\log _{10} \mathrm{IU} / \mathrm{ml} \|$ & (N=363) & (N=364) & (N=361) \\
HCV RNA $\geq 800,000 \mathrm{IU} / \mathrm{ml}$ - no. (\%) & $6.3 \pm 0.7$ & $6.3 \pm 0.7$ & $6.3 \pm 0.7$ \\
Stage of fibrosis and cirrhosis - no. (\%) & $281(77)$ & $279(77)$ & $279(77)$ \\
$\quad$ None or minimal fibrosis & & & $147(41)$ \\
$\quad$ Portal fibrosis & $134(37)$ & $128(35)$ & $141(39)$ \\
$\quad$ Bridging fibrosis & $156(43)$ & $151(41)$ & $52(14)$ \\
$\quad$ Cirrhosis & $52(14)$ & $59(16)$ & $21(6)$ \\
\hline
\end{tabular}

* Plus-minus values are means \pm SD. The PR (control) group received telaprevir-matched placebo plus peginterferon alfa$2 \mathrm{a}$ and ribavirin for the first 12 weeks followed by peginterferon-ribavirin for 36 weeks. The telaprevir groups received telaprevir for 8 weeks (T8PR) or 12 weeks (T12PR), as well as peginterferon-ribavirin for a total of 24 weeks or 48 weeks. There were no significant differences in the listed characteristics among the telaprevir groups and the control group, except for body-mass index, for which there was a significant difference between the T12PR group and the PR group $(\mathrm{P}=0.02)$.

$\dagger$ The body-mass index is the weight in kilograms divided by the square of the height in meters.

Race and ethnic group were self-reported and were not mutually exclusive.

$\int$ To convert the values for total bilirubin to milligrams per deciliter, divide by 17.1.

9 Hepatitis $\mathrm{C}$ virus (HCV) genotype and subtype were determined with the use of the VERSANT HCV genotype 2.0 assay (INNO-LiPA, Innogenetics).

| HCV RNA levels were measured with the use of COBAS TaqMan HCV assay (Roche), which has a lower limit of quantification of 25 IU per milliliter and a lower limit of detection of $10 \mathrm{IU}$ per milliliter.

discontinuation of telaprevir (or placebo) only owing to adverse events was higher among the patients who received telaprevir than among those who received placebo (11\% in the T12PR group and $7 \%$ in the T8PR group, vs. $1 \%$ in the PR group). Anemia and rash were the most frequently reported adverse events that led to the discontinuation of telaprevir-based regimens. A total of $7 \%$ of the patients in the T12PR group and 5\% in the T8PR group discontinued telaprevir owing to rash, whereas $1.4 \%$ and $0.5 \%$ in the two groups, respectively, discontinued all treatment owing to rash during the telaprevir (or placebo) phase of the study. Rashes were primarily eczematous and were reversible with discontinuation of telaprevir. One case of the Stevens-Johnson syndrome occurred approximately 11 weeks after the last dose of telaprevir had been administered.

More patients in the telaprevir groups than in the PR group discontinued all treatment owing to anemia (1\% in the T12PR group and 3\% in the T8PR group, vs. $<1 \%$ in the PR group), and $4 \%, 2 \%$, and $0 \%$ of the patients in the three groups, respectively, discontinued telaprevir (or placebo) only. A total of 17 patients in the T12PR group, 17 in the T8PR group, and 6 in the PR group received blood transfusions during the study. The decrease in hemoglobin levels was more pronounced in patients receiving telaprevirbased regimens than in patients in the PR group and was reversed with the discontinuation of telaprevir (Fig. 3 in the Supplementary Appendix). The largest difference in mean hemoglobin levels between the T12PR group and the PR group (1.04 g per deciliter lower in the T12PR group) and between the T8PR group and the PR group $(1.11 \mathrm{~g}$ per deciliter lower in the T8PR group) was observed at week 8 of treatment.

Four deaths occurred during the study: three after all study drugs had been discontinued and one (in the PR group) during the treatment phase. Two patients - one in the T12PR group and one in the PR group - died as a result of suicide, one patient in the T12PR group died from HCV infection and liver disease, and one in the T8PR group died from an unknown cause.

\section{DISCUSSION}

These results confirm earlier studies and showed a significant increase in the rate of sustained virologic response among patients with HCV genotype 1 infection who are treated with a regimen combining peginterferon alfa-2a and ribavirin with tela- 


\begin{tabular}{|c|c|c|c|}
\hline Response & $\begin{array}{c}\mathrm{Tl2PR} \\
(\mathrm{N}=363)\end{array}$ & $\begin{array}{c}\text { T8PR } \\
(\mathrm{N}=364)\end{array}$ & $\begin{array}{c}P R \\
(N=361)\end{array}$ \\
\hline \multicolumn{4}{|l|}{ Undetectable HCV RNA during treatment period — no. (\%)* } \\
\hline At week 4 & $246(68)$ & $242(66)$ & $34(9)$ \\
\hline At weeks 4 and 12 & $212(58)$ & $207(57)$ & $29(8)$ \\
\hline Undetectable HCV RNA at end of treatment period - no. (\%) & $314(87)$ & $295(81)$ & $229(63)$ \\
\hline \multicolumn{4}{|l|}{$\begin{array}{l}\text { Undetectable HCV RNA } 24 \text { wk after end of treatment: sustained } \\
\text { virologic response }- \text { no./total no. (\%) } \dagger\end{array}$} \\
\hline All patientsł & $271 / 363(75)$ & $250 / 364(69)$ & $158 / 361(44)$ \\
\hline Patients with undetectable HCV RNA at weeks 4 and 12 & $189 / 212(89)$ & $171 / 207(83)$ & $28 / 29(97)$ \\
\hline Patients with detectable HCV RNA at weeks 4 or week 12 & $82 / 151(54)$ & $79 / 157(50)$ & $130 / 332(39)$ \\
\hline Patients with undetectable HCV RNA at week 4 & $206 / 246(84)$ & $188 / 242(78)$ & $32 / 34(94)$ \\
\hline Patients with detectable HCV RNA at week 4 & $65 / 117(56)$ & $62 / 122(51)$ & $126 / 327(39)$ \\
\hline Undetectable HCV RNA at 72 wk - no. (\%) $\int$ & $265(73)$ & $243(67)$ & $158(44)$ \\
\hline \multicolumn{4}{|l|}{$\begin{array}{l}\text { Relapse among patients with undetectable HCV RNA at end of } \\
\text { treatment period - no./total no. (\%) }\end{array}$} \\
\hline All patients & $27 / 314(9)$ & $28 / 295(9)$ & $64 / 229(28)$ \\
\hline Patients who completed treatment & $17 / 264(6)$ & $18 / 247(7)$ & $51 / 189(27)$ \\
\hline
\end{tabular}

* Patients with undetectable HCV RNA at week 4 met the criterion for a rapid virologic response, and patients with undetectable HCV RNA at weeks 4 and 12 met the criterion for an extended rapid virologic response.

$\dagger$ Sustained virologic response (undetectable HCV RNA 24 weeks after the end of treatment) was the primary end point.

$t$ All patients who received at least one dose of study drug were included in the analysis. The difference in response rates was 31 percentage points ( $95 \%$ confidence interval $[\mathrm{Cl}], 24$ to 38 ) between the T12PR and PR groups and 25 percentage points $(95 \% \mathrm{Cl}, 18$ to 32$)$ between the T8PR and PR groups.

$\int$ The 72-week assessment was performed 24 weeks after the end of treatment in patients who received 48 weeks of treatment and 48 weeks after end of treatment in patients who received 24 weeks of treatment.

previr for 12 or 8 weeks, followed by peginterferonribavirin alone, for a total of 24 or 48 weeks of therapy, as compared with a standard regimen of peginterferon-ribavirin alone for 48 weeks. Among patients with $\mathrm{HCV}$ genotype 1 infection who have not previously received treatment, the potential to shorten the duration of therapy with peginterferon-ribavirin to less than 48 weeks without impairing the chance of a sustained virologic response is currently limited to the small number of patients with a low viral load who have undetectable HCV RNA at week 4. ${ }^{12-15}$ In contrast, in the current study, more than half the patients who received telaprevir had undetectable HCV RNA at weeks 4 and 12, indicating an extended rapid virologic response, and relapse occurred infrequently in these patients after 24 weeks of treatment, suggesting that a total treatment duration of 24 weeks is sufficient for these patients. A longer duration of peginterferon-ribavirin therapy is indicated for patients who do not have an extended rapid virologic response.
Patients in the T8PR group, as compared with those in the T12PR group, had a lower rate of response, and also a slightly lower rate of discontinuation of telaprevir. The lower rate of virologic failure during treatment in the T12PR group as compared with the T8PR group and the more frequent emergence of wild-type and lower-level resistant variants beyond week 12 in the T8PR group than in the T12PR group are probably attributable to more efficient elimination of these viral strains as a result of the additional 4 weeks of telaprevir therapy in the T12PR group.

The rates of sustained virologic response were substantially improved with the addition of telaprevir in patients with negative predictive factors for a response to peginterferon-ribavirin treatment, such as bridging fibrosis or cirrhosis, older age, diabetes, and HCV RNA levels of 800,000 IU per milliliter or more. An increase in sustained virologic response by a factor of more than 2 occurred with telaprevir in black patients, in whom low response rates to interferon have been re- 


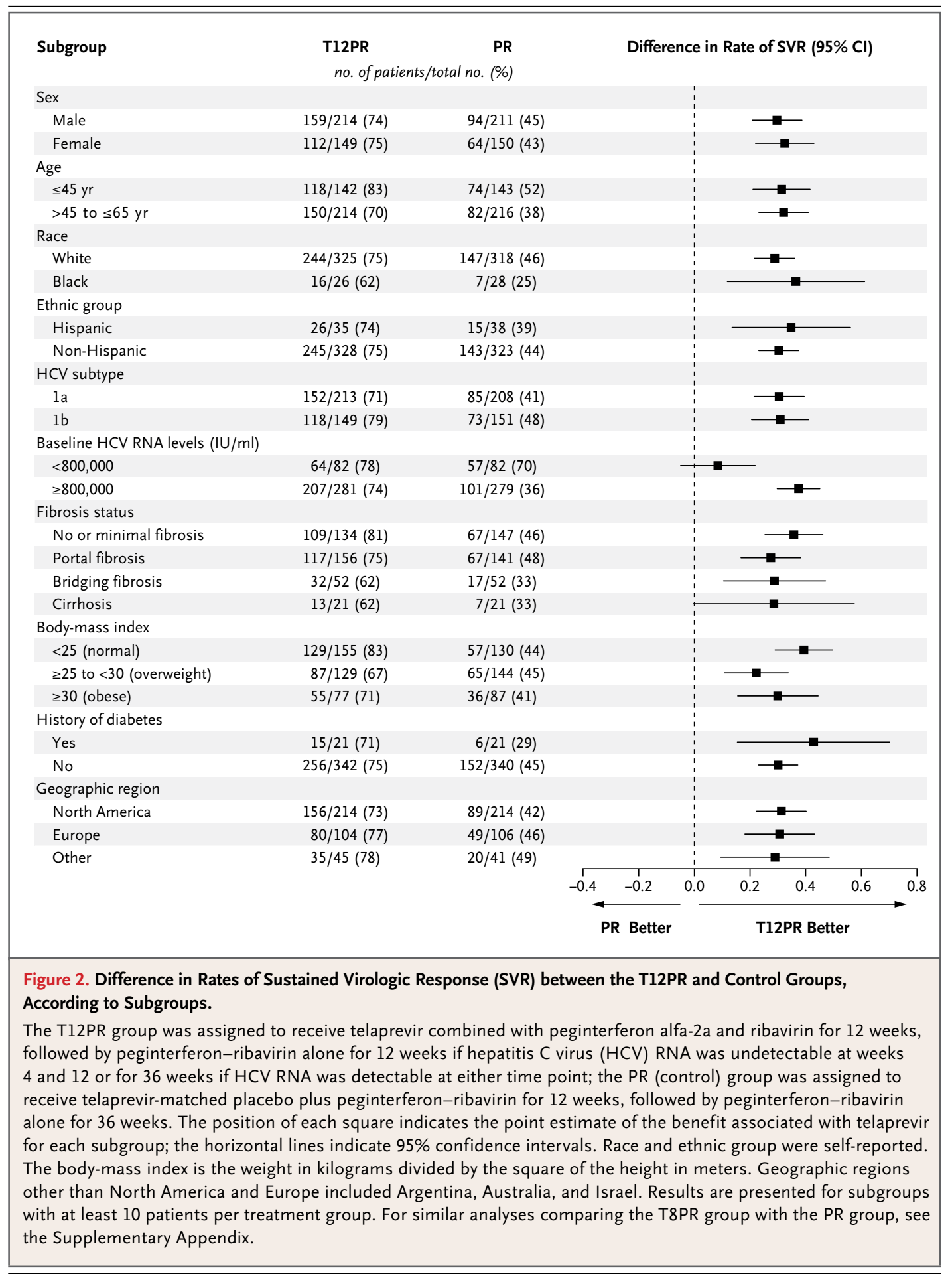

ported. ${ }^{16-19}$ This finding has been associated with a high prevalence in black persons of the $\mathrm{T}$ allele at the rs1297860 locus in the region of the IL28B gene. ${ }^{20,21}$ Additional studies are needed to clarify the relationship between IL28B polymorphisms and the response to telaprevir or other directacting antiviral agents.

As suggested previously, telaprevir, as compared with peginterferon-ribavirin alone, was associated with a higher incidence of adverse events such 


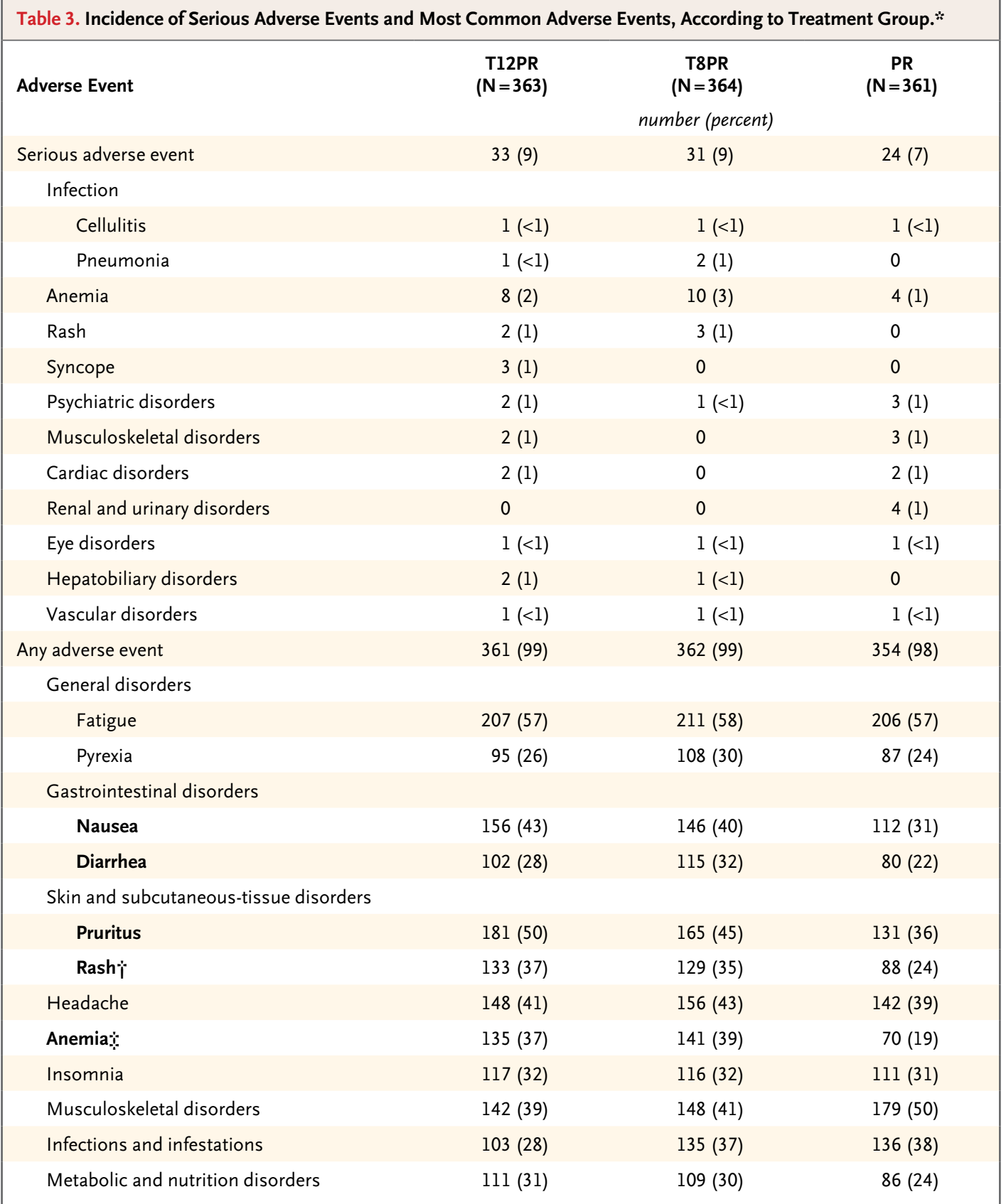

* The serious adverse events listed here are those that were reported in at least three patients during the treatment period, and the other adverse events listed are those that were reported during the treatment period in at least $30 \%$ of patients in at least one of the three study groups; all grades of events are included. Other common adverse events, including anorectal symptoms, are shown in Table 1 in the Supplementary Appendix. Adverse events in bold are those that occurred at an incidence that was 10 percentage points higher in either group receiving telaprevir than in the PR (control) group. $\dagger$ As assessed with the use of a group of related terms to identify all dermatologic events, the incidences of rash events and grade 3 rash events were $61 \%$ and $6 \%$, respectively, in the T12PR group, $58 \%$ and $4 \%$, respectively, in the T8PR group, and $48 \%$ and $1 \%$, respectively, in the PR group during the overall treatment phase.

$t$ The percentages of patients with hemoglobin levels of less than $10 \mathrm{~g}$ per deciliter and less than $8.5 \mathrm{~g}$ per deciliter were $36 \%$ and $9 \%$, respectively, in the T12PR group, $40 \%$ and $9 \%$, respectively, in the T8PR group, and $14 \%$ and $2 \%$, respectively, in the PR group. 
as rash, gastrointestinal disorders, and anemia..$^{8-10}$ In this study, rashes resolved with the discontinuation of telaprevir; 7 to $11 \%$ of patients discontinued telaprevir, and only 0.5 to $1.4 \%$ discontinued all therapy owing to rash. The implementation of managed, sequential discontinuation of medications for severe rash may have led to the lower rate of overall discontinuation of treatment in this study. Anemia led to the discontinuation of treatment in few patients.

In conclusion, telaprevir-containing regimens, as compared with a regimen of peginterferonribavirin alone, were associated with a significant increase in the rates of sustained virologic response, overall and in all the subgroups of patients that were analyzed. The majority of patients who were treated with telaprevir had undetectable HCV RNA at weeks 4 and 12 and received only 24 weeks of total therapy. Numerically higher response rates, with a small increment in reversible adverse events, were observed with a regimen of 12 weeks, as compared with 8 weeks, of telaprevir combined with peginterferon-ribavirin, followed by additional weeks of peginterferon-ribavirin alone. The significant improvement in the rates of sustained virologic response with telaprevir-based therapy and the capacity for response-guided therapy to shorten the duration of exposure to peginterferonribavirin among patients who have a rapid response represent important advances in the treatment of patients with HCV genotype 1 infection.

Supported by Vertex Pharmaceuticals and Tibotec.

Dr. Jacobson reports receiving consulting fees from Abbott, Achillion Pharmaceuticals, Anadys Pharmaceuticals, Biolex Therapeutics, Boehringer Ingelheim, Bristol-Myers Squibb, Gilead Sciences, GlaxoSmithKline, GlobeImmune, Human Genome Sciences, Novartis, Pharmasset, Pfizer, Roche-Genentech, Sanofi-Aventis, Schering-Plough (now part of Merck), Tibotec, Vertex Pharmaceuticals, and ZymoGenetics, lecture fees from Bristol-Myers Squibb, Gilead Sciences, Roche-Genentech, and Schering-Plough (Merck), and grant support from Anadys Pharmaceuticals, Boehringer Ingelheim, Bristol-Myers Squibb, Gilead Sciences, GlobeImmune, Human Genome Sciences, Novartis, Pfizer, Pharmasset, Roche-Genentech, Schering-Plough (Merck), Tibotec, Vertex Pharmaceuticals, and ZymoGenetics; Dr. McHutchison, being an employee of Gilead Sciences, participating in advisory committees or review panels for Abbott, Anadys Pharmaceuticals, Biolex, Gilead Sciences, National Genetics Institute, Pharmasset, Pfizer, United Therapeutics, and Vertex Pharmaceuticals, and receiving grant support from GlaxoSmithKline, GlobeImmune, Human Genome Sciences, Idera Pharmaceuticals, Intarcia Therapeutics, Medtronics, Novartis, Osiris Therapeutics, Roche-Genentech, Schering-Plough (Merck), and Vertex Pharmaceuticals; Dr. Dusheiko, receiving consulting fees from Abbott, Boehringer Ingelheim, Bristol-Myers Squibb, GlaxoSmithKline, Human Genome Sciences, Novartis, Pharmasset, Pfizer, RocheGenentech, Schering-Plough (Merck), Tibotec, Vertex Pharmaceu- ticals, and ZymoGenetics, travel support from Gilead Sciences, and grant support from Gilead Sciences, Novartis, Pharmasset, Hoffmann-La Roche, Schering-Plough (Merck), Tibotec, and Vertex Pharmaceuticals; Dr. Di Bisceglie, receiving consulting fees from Abbott, Anadys Pharmaceuticals, Bristol-Myers Squibb, GlobeImmune, Idenix Pharmaceuticals, Pharmasset, RocheGenentech, and Vertex Pharmaceuticals, and grant support from Bristol-Myers Squibb, Gilead Sciences, GlobeImmune, Idenix Pharmaceuticals, and Roche-Genentech; Dr. Reddy, receiving consulting fees from Human Genome Sciences, Roche-Genentech, Salix Pharmaceuticals, Schering-Plough (Merck), Tibotec, and Vertex Pharmaceuticals and grant support from Bristol-Myers Squibb, Gilead Sciences, Roche-Genentech, Schering-Plough (Merck), Tibotec, and Vertex Pharmaceuticals; Dr. Bzowej, receiving consulting fees from Gilead Sciences and Vertex Pharmaceuticals and grant support from Anadys Pharmaceuticals, Bristol-Myers Squibb, Gilead Sciences, GlaxoSmithKline, Human Genome Sciences, Idenix Pharmaceuticals, Johnson \& Johnson, LifeCycle Pharma, Pharmasset, Roche-Genentech, ScheringPlough (Merck), Tibotec, and Vertex Pharmaceuticals; Dr. Marcellin, receiving consulting fees from Abbott, Biolex Therapeutics, Boehringer Ingelheim, Bristol-Myers Squibb, Gilead Sciences, InterMune, MSD Pharmaceuticals (Merck), Novartis, Pfizer, Pharmasset, Roche-Genentech, Schering-Plough (Merck), Tibotec, Vertex Pharmaceuticals, and ZymoGenetics, lecture fees from Bristol-Myers Squibb, Gilead Sciences, InterMune, Novartis, Roche-Genentech, Schering-Plough (Merck), and Tibotec, and grant support from Echosens, Gilead Sciences, Roche-Genentech, and Schering-Plough (Merck); Dr. Muir, receiving consulting fees from Bristol-Myers Squibb, Pharmasset, Santaris Pharma, Schering-Plough (Merck), Vertex Pharmaceuticals, and ZymoGenetics and grant support from Anadys Pharmaceuticals, Boehringer Ingelheim, Gilead Sciences, GlaxoSmithKline, Idera Pharmaceuticals, Medtronic, Pfizer, Pharmasset, Santaris Pharma, Scynexis, Three Rivers Pharmaceuticals, Schering-Plough (Merck), and Vertex Pharmaceuticals; Dr. Ferenci, receiving consulting fees from Achillion Pharmaceuticals, Boehringer Ingelheim, MSD Pharmaceuticals (Merck), Novartis, Pfizer, Rottapharm Madaus, Roche-Genentech, Tibotec, and Vertex Pharmaceuticals and grant support from Roche-Genentech; Dr. Flisiak, receiving consulting fees from Bristol-Myers Squibb, Debiopharm, Human Genome Sciences, Novartis, Pfizer, Roche, and ScheringPlough (Merck) and lecture fees from Bristol-Myers Squibb, Gilead Sciences, GlaxoSmithKline, Roche, and Schering-Plough (Merck); Dr. J. George, receiving consulting fees from Bristol-Myers Squibb, Gilead Sciences, MSD Pharmaceuticals (Merck), and Roche-Genentech, lecture fees from Bristol-Myers Squibb, Gilead Sciences, MSD Pharmaceuticals (Merck), Novartis, and RocheGenentech, and grant support from MSD Pharmaceuticals (Merck) and Roche-Genentech; Dr. Rizzetto, receiving consulting fees from Bristol-Myers Squibb, Janssen-Cilag, Roche-Genentech and Schering-Plough (Merck) and lecture fees from Bristol-Myers Squibb, Gilead Sciences, GlaxoSmithKline, Roche-Genentech, and Schering-Plough (Merck); Dr. Shouval, receiving consulting fees from SciGen and Biotest, lecture fees from Cangene, Gilead Sciences, Roche-Genentech, and Schering-Plough (Merck), and grant support from Human Genome Sciences, Novartis, Pfizer, RocheGenentech, and Schering-Plough (Merck); Dr. Sola, receiving consulting fees from Bristol-Myers Squibb, Gilead Sciences, Novartis, Roche-Genentech, and Tibotec-Janssen-Cilag, lecture fees from Bristol-Myers Squibb, Gilead Sciences, Novartis, and RocheGenentech, and grant support from Gilead Sciences, RocheGenentech, and Schering-Plough (Merck); Dr. Terg, receiving grant support from Fundación CIDEA; Dr. Yoshida, receiving consulting fees from Cangene, Gilead Sciences, Roche-Genentech, and Schering-Plough (Merck), lecture fees from Vertex Pharmaceuticals, and grant support from Boehringer Ingelheim, Cangene, 
Gilead Sciences, Human Genome Sciences, Novartis, Pfizer, Roche-Genentech, Schering-Plough (Merck), Tibotec, and Vertex Pharmaceuticals; L. Bengtsson and Drs. Adda, Sankoh, Kieffer, S. George, and Kauffman, being employees of and owning stock in Vertex Pharmaceuticals; and Dr. Zeuzem, receiving consulting fees from Abbott, Achillion Pharmaceuticals, Anadys Pharmaceuticals, Bayer, Boehringer Ingelheim, Bristol-Myers Squibb, Gilead Sciences, Human Genome Sciences, iTherX, Novartis, Pfizer, Pharmasset, Roche-Genentech, Santaris Pharma, ScheringPlough (Merck), Tibotec, and Vertex Pharmaceuticals and lecture fees from Bristol-Myers Squibb, Gilead Sciences, Roche-Genentech, and Schering-Plough (Merck). No other potential conflict of interest relevant to this article was reported.

Disclosure forms provided by the authors are available with the full text of this article at NEJM.org.
We thank the study coordinators, nurses, and patients involved in the study; the personnel at the coordinating center for the study, including Eric Zafarana, M.D. (Parexel), and Andrew J. Muir, M.D. (Duke Clinical Research Institute), for medical monitoring, Hans Tillman, M.D. (Duke Clinical Research Institute), for viral monitoring, and Ann-Marie Dunne, M.P.H. (Vertex Pharmaceuticals), for clinical project management; John Alam, M.D., and Lindsay McNair, M.D. (both former employees of Vertex Pharmaceuticals), for contribution to the design of the study; and Xiangchen Cui, Ph.D., Claude Fiset, and Min Chen, Ph.D., for statistical programming, Doug Bartels, Ph.D., and Ann Kwong, Ph.D., for virology analysis, Angela Scott, Ph.D., for data analysis, and Valérie Philippon, Ph.D., for medical writing, editorial assistance, and coordination support (all from Vertex Pharmaceuticals).
REFERENCES

1. Di Bisceglie AM, Lyra AC, Schwartz $\mathrm{M}$, et al. Hepatitis C-related hepatocellular carcinoma in the United States: influence of ethnic status. Am J Gastroenterol 2003;98:2060-3.

2. World Health Organization. Global surveillance and control of hepatitis C: report of a WHO consultation organized in collaboration with the Viral Hepatitis Prevention Board, Antwerp, Belgium. J Viral Hepat 1999;6:35-47.

3. El Serag HB, Mason AC. Rising incidence of hepatocellular carcinoma in the United States. N Engl J Med 1999;340:74550.

4. Thomas MB, Jaffe D, Choti MM, et al. Hepatocellular carcinoma: consensus recommendations of the National Cancer Institute trials planning meeting. $\mathrm{J}$ Clin Oncol 2010;28:3994-4005. [Erratum, J Clin Oncol 2010;28:5350.]

5. Manns M, McHutchison JG, Gordon $\mathrm{SC}$, et al. Peginterferon alfa-2b plus ribavirin compared with interferon alfa-2b plus ribavirin for initial treatment of chronic hepatitis C: a randomized trial. Lancet 2001;358:958-65.

6. Fried MW, Shiffman ML, Reddy KR, et al. Peginterferon alfa-2a plus ribavirin for chronic hepatitis $\mathrm{C}$ virus infection. N Engl J Med 2002;347:975-82.

7. McHutchison JG, Lawitz EJ, Shiffman $M L$, et al. Peginterferon alfa- $2 b$ or alfa- $2 a$ with ribavirin for treatment of hepatitis $\mathrm{C}$ infection. N Engl J Med 2009;361:580-93. [Erratum, N Engl J Med 2009;361:1027.]
8. McHutchison JG, Everson GT, Gordon SC, et al. Telaprevir with peginterferon and ribavirin for chronic HCV genotype 1 infection. N Engl J Med 2009;360:1827-38. [Erratum, N Engl J Med 2009:361:1516.]

9. Hézode C, Forestier N, Dusheiko G, et al. Telaprevir and peginterferon with or without ribavirin for chronic HCV infection. N Engl J Med 2009;360:1839-50.

10. McHutchison JG, Manns MP, Muir AJ et al. Telaprevir for previously treated chronic HCV infection. N Engl J Med 2010, 362:1292-303. [Erratum, N Engl J Med 2010;362:1647.

11. Kieffer TL, Sarrazin C, Miller JS, et al. Telaprevir and pegylated interferon-alpha2a inhibit wild-type and resistant genotype 1 hepatitis $\mathrm{C}$ virus replication in patients. Hepatology 2007;46:631-9.

12. Zeuzem S, Buti M, Ferenci P, et al. Efficacy of 24 weeks treatment with peginterferon alfa-2b plus ribavirin in patients with chronic hepatitis $C$ infected with genotype 1 and low pretreatment viremia. J Hepatol 2006;44:97-103.

13. Jensen DM, Morgan TR, Marcellin P, et al. Early identification of HCV genotype 1 patients responding to 24 weeks peginterferon alpha-2a $(40 \mathrm{kd}) /$ ribavirin therapy. Hepatology 2006;43:954-60. [Erratum, Hepatology 2006;43:1410.]

14. Mangia A, Minerva N, Bacca D, et al. Individualized treatment duration for hepatitis $\mathrm{C}$ genotype 1 patients: a randomized controlled trial. Hepatology 2008;47:4350 .
15. Moreno C, Deltenre P, Pawlotsky JM, Henrion J, Adler M, Mathurin P. Shortened treatment duration in treatmentnaïve genotype $1 \mathrm{HCV}$ patients with rapid virological response: a meta-analysis. J Hepatol 2010;52:25-31.

16. Jeffers LJ, Cassidy W, Howell CD, Hu $\mathrm{S}$, Reddy KR. Peginterferon alfa-2a (40 kd) and ribavirin for black American patients with chronic HCV genotype 1 . Hepatology 2004;39:1702-8.

17. Conjeevaram HS, Fried MW, Jeffers LJ, et al. Peginterferon and ribavirin treatment in African American and Caucasian American patients with hepatitis C genotype 1. Gastroenterology 2006;131:470-7. 18. Muir AJ, Bornstein JD, Killenberg PG. Peginterferon alfa-2b and ribavirin for the treatment of chronic hepatitis $\mathrm{C}$ in blacks and non-Hispanic whites. N Engl J Med 2004;350:2265-71. [Erratum, N Engl J Med 2004:351:1268.]

19. Jacobson IM, Brown RS Jr, McCone J, et al. Impact of weight-based ribavirin with peginterferon alfa-2b in African Americans with hepatitis C virus genotype 1 . Hepatology 2007;46:982-90.

20. Ge D, Fellay J, Thompson AJ, et al. Genetic variation in IL28B predicts hepatitis C treatment-induced viral clearance. Nature 2009;461:399-401.

21. Thomas DL, Thio CL, Martin MP, et al. Genetic variation in IL28B and spontaneous clearance of hepatitis $\mathrm{C}$ virus. Nature 2009;461:798-801.

Copyright $\odot 2011$ Massachusetts Medical Society. 